\title{
Molecular Mechanisms for the Inflammation-Resolving Actions of Lenabasum
}

\author{
Sumner Burstein \\ Department of Biochemistry and Molecular Pharmacology, University of Massachusetts Medical School, Worcester, \\ Massachusetts
}

Received June 9, 2020; accepted November 19, 2020

\section{ABSTRACT}

A first-in-class cannabinoid analog called lenabasum that is a CB2 agonist is being developed as an inflammation-resolving drug candidate. Thus far, specific therapeutic targets include scleroderma, cystic fibrosis, dermatomyositis, and lupus, all of which represent unmet medical needs. Two somewhatindependent molecular mechanisms for this type of action are here proposed. Both pathways initially involve the release of free arachidonic acid after activation of the CB2 receptor and phospholipase A2 by lenabasum. The pathways then diverge into a cyclooxygenase 2-mediated and a lipoxygenasemediated route. The former leads to increased levels of the cyclopentenone prostaglandin 15-deoxy- $\Delta^{12,14}$-prostaglandin$\mathrm{J}_{2}$ that can activate the NLPR3 inflammasome, which in turn releases caspase-3, leading to apoptosis and the resolution of chronic inflammation. The lipoxygenase-mediated pathway stimulates the production of lipoxin $\mathrm{A}_{4}$ as well as other signaling molecules called specialized proresolving mediators. These also have inflammation-resolving actions. It is not well understood under which conditions each of these mechanisms operates and whether there is crosstalk between them. Thus, much remains to be learned about the mechanisms describing the actions of lenabasum

\section{SIGNIFICANCE STATEMENT}

The resolution of chronic inflammation is a major unmet medical need. The synthetic nonpsychoactive cannabinoid lenabasum could provide a safe and effective drug for this purpose. Two putative molecular mechanisms are suggested to better understand how lenabasum produces this action. In both, different metabolites of arachidonic acid act as mediators.

\section{Introduction}

The effects of cannabinoids on the eicosanoid family of signaling molecules have been under investigation for some time (Burstein, 2019). Among the compounds studied were the phytocannabinoids tetrahydrocannabinol (THC) and cannabidiol. In addition, synthetic agents, such as ajulemic acid (also called anabasum or lenabasum), CP55,940, JWH-133, and WIN-55,212-2, were reported on. The actions studied were alteration of behavioral responses, reduction of pain sensation, resolution of inflammation, hypotensive and vasorelaxant responses, anticancer activity, and reduction of intraocular pressure. Thus, there appear to be a number of cannabinoid and eicosanoid interactions that could be exploited for therapeutic

This paper was produced without funding support from any source.

Conflicts: The author is a cofounder but not an employee of Corbus Pharmaceuticals and owns stock in the company.

https://doi.org/10.1124/molpharm.120.000083. purposes (Burstein, 2019). In the example of lenabasum, these will be discussed below, and a brief history of its development now follows (Table 1).

The events leading to the discovery and development of lenabasum began with investigations into the metabolism of the cannabinoids (Burstein et al., 1972). It was concluded from these studies that the terminal metabolite of THC is THC-11oic acid. Interestingly, it was observed that this metabolite unlike THC was not psychoactive; however, it appeared to have anti-inflammatory activity (Burstein et al., unpublished data). This observation was exploited for the purpose of finding the drug candidate ajulemic acid (Fig. 1) that was subsequently reported (Burstein et al., 1992) and later named lenabasum. Lenabasum is the 9-carbon 1,1-dimethylheptyl side-chain analog of THC-11-oic acid. In this study, it was shown that lenabasum had potent activity in the mouse pawedema assay (est. $\mathrm{ED}_{50} ; 0.025 \mathrm{mg} / \mathrm{kg}$ ) and in the mouse hotplate assay (est. $\mathrm{ED}_{50} ; 0.10 \mathrm{mg} / \mathrm{kg}$ ). In the hot-plate assay, lipoxin $\mathrm{A}_{4}$; $\mathrm{LXB}_{4}$, lipoxin $\mathrm{B}_{4}$; LOX, lipoxygenase; $\mathrm{NF} \kappa \mathrm{B}$, nuclear factor $\kappa \mathrm{B}$; NSAID, nonsteroidal anti-inflammatory drug; PG, prostaglandin; PGJ, 15-deoxy- $\Delta^{12,14}$-prostaglandin-J $\mathrm{J}_{2}$; PLA2, phospholipase A2; PPAR $\gamma$, peroxisome proliferator-activated receptor $\gamma$; Rv, resolvin; SPM, specialized proresolving mediator; THC, tetrahydrocannabinol; TNF $\alpha$, tumor necrosis factor $\alpha$. 
TABLE 1

Reduction of IL- $1 \beta$ secretion by lenabasum ${ }^{a}$ Adapted from (Zurier and Burstein, 2016)

\begin{tabular}{lc}
\hline Dose $(\mathrm{mg}$ by mouth) & \% Reduction IL-1 $\beta^{b}$ \\
\hline 3 & 14.6 \\
6 & 35.6 \\
10 & 47.4 \\
\hline
\end{tabular}

${ }^{a}$ Cells were stimulated peripheral blood monocytes from healthy volunteers administered oral lenabasum.

${ }^{b}$ Mean percent reduction in three subjects $5 \mathrm{~h}$ after lenabasum administration.

a "bell-shaped" curve was seen, suggesting a different mechanism than in the paw-edema assay. In a later preclinical report, this idea was confirmed, and it was concluded that "[a]vailable evidence indicates that CT-3 (lenabasum) exhibits two distinct pharmacological properties: an anti-inflammatory property occurring at a very low dose and an analgesic property occurring at a higher dose" (Dajani et al., 1999).

Since 1992 several studies have confirmed and expanded the therapeutic properties of lenabasum. Thus, adjuvant-induced polyarthritis in rats was used as a model of chronic inflammation and joint tissue injury (Zurier et al., 1998). Histopathologic studies of tibiotarsal joints showed that lenabasum treatment $(0.1 \mathrm{mg} / \mathrm{kg}$ three times per week) reduced pannus formation and joint tissue injury by about $80 \%$ when compared with vehicle-treated rats. A subsequent study also in rats further confirmed the analgesic actions of lenabasum and, additionally, showed that it did not alter motor function in the Rotarod procedure at $4.64 \mathrm{mg} / \mathrm{kg}$ i.v., the latter being a desirable property for a drug candidate (Burstein et al., 1998).

Because of its potent effects on cell adhesion (Burstein et al., 1992), it was decided that lenabasum would be tested in a cancer model for possible inhibition of metastatic disease. Lenabasum administered by mouth to nude mice at a dosage several orders of magnitude below that which produces toxicity inhibited the growth of subcutaneously implanted U87 human glioma cells (Recht et al., 2001). It was shown that lenabasum can produce significant antitumor activity, and its effects are mediated by the action of CB2 receptors. A dramatic decrease in ascites in treated animals suggested concurrent antiinflammatory and antimetastatic action (unpublished data).

Interleukin (IL)- $1 \beta$ and tumor necrosis factor $\alpha(\mathrm{TNF} \alpha)$ are critical factors in the progression of inflammation and joint tissue injury in patients with rheumatoid arthritis. Thus, the effects of lenabasum in peripheral blood and synovial fluid monocytes on these factors in samples from both normal and rheumatoid arthritis subjects were examined (Zurier et al., 2003). Cells were treated with lenabasum $(0-30 \mu \mathrm{M})$ in vitro and then stimulated with lipopolysaccharide. Drug treatment suppression was maximal $(50.4 \%)$ at $10 \mu \mathrm{M}(P<0.05$ vs. untreated controls, $N=7$ ).
Studies were done to provide some insight into the mechanism of the anti-inflammatory action of lenabasum (Bidinger et al., 2003). Thus, apoptosis resulting from lenabasum treatment of human $\mathrm{T}$ cells in vitro was determined by measuring annexin $\mathrm{V}$ expression, caspase-3 activity, DNA fragmentation, and microscopy. Lenabasum induced apoptosis of $\mathrm{T}$ cells in a dose- and time-dependent manner. Incubation of cells with $10 \mu \mathrm{M}$ lenabasum resulted in significant increases (2.5- to 8.4-fold) in annexin V-positive cells. Apoptosis preceded loss of cell viability as shown by trypan blue dye exclusion, confirming that cell loss was due to programmed cell death rather than necrosis.

A pharmacokinetic analysis showed that lenabasum has limited brain penetration, with a brain/plasma ratio of 0.4 after oral administration (Dyson et al., 2005). This suggests that this is the basis for the lack of psychoactivity rather than a higher affinity for CB2 versus CB1. In contrast with other reports, it was found that lenabasum binds to human cannabinoid receptors in vitro with high affinity at hCB1 $(\mathrm{Ki}=6 \mathrm{nM})$ and hCB2 $(\mathrm{Ki}=56 \mathrm{nM})$. The sample of compound used was prepared, and no purity data were provided, raising the possibility of impurities with high affinity for CB1 receptors. The likelihood of such a possibility has been demonstrated more recently (Tepper et al., 2014).

Analgesic actions of lenabasum in animal models of neuropathic and inflammatory pain have been reported (Mitchell et al., 2005). It was compared with HU-210, and both were found to be effective. However, lenabasum, unlike HU-210, did not exhibit any of the motor side effects associated with THC and other nonselective cannabinoid receptor agonists. This again suggests a lack of central nervous system activity by lenabasum.

The reduction by lenabasum of urinary frequency induced by nociceptive stimuli in the bladder has been observed in rats (Hiragata et al., 2007). It was effective when given intravenously at a dose $10 \mathrm{mg} / \mathrm{kg}$. Evidence was presented supporting a mechanism involving CB1 rather than CB2, which is proposed for inflammation-resolving action. It was suggested that lenabasum could be effective in the treatment of patients with painful bladder syndrome or interstitial cystitis.

Unlike the traditional anti-inflammatory agents [e.g., nonsteroidal anti-inflammatory drugs (NSAIDs)] that inhibit the production of eicosanoids, lenabasum stimulates their production through the arachidonic acid cascade (Stebulis et al., 2008). However, the difference is in the specific eicosanoids involved. Lenabasum treatment results in the elevation of the inflammation-resolving molecule prostaglandin (PG) $\mathrm{D}_{2}$ and the resulting 15-deoxy- $\Delta^{12,14}$-prostaglandin- $\mathrm{J}_{2}$ (PGJ) series that promotes the resolution of inflammation. The NSAIDs generally act by inhibiting the production of the proinflammatory eicosanoid $\mathrm{PGE}_{2}$. In the study cited here, the effect of lenabasum on cyclooxygenase (COX)-2 expression and eicosanoid production
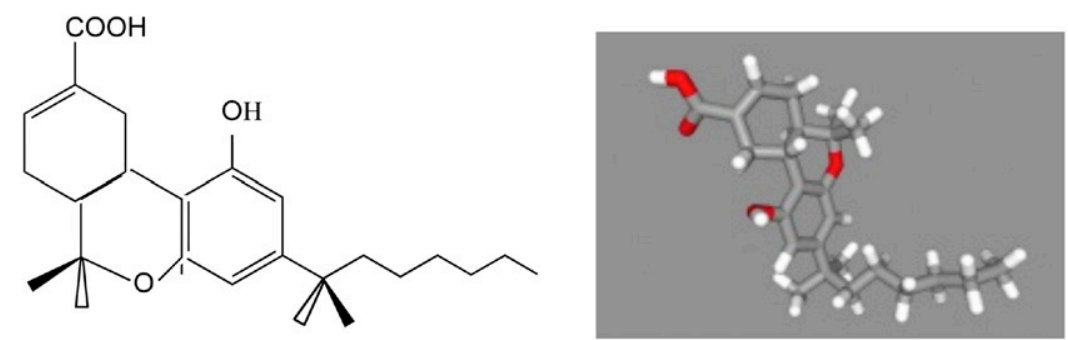

Fig. 1. Chemical structure of lenabasum (also called ajulemic acid). Systematic name: (6ar,10ar)-3-(1,1-dimethylheptyl)1-hydroxy-6,6-dimethyl-6a,7,10,10a tetrahydro-6h-benzo[c] chromene-9-carboxylic acid. Molecular mass $=400.5 \mathrm{Da}$. Color code: carbon, gray; hydrogen, white; oxygen, red. 
in human fibroblast-like synovial (FLS) cells was measured. Treatment of FLS cells with lenabasum increased $15 \mathrm{~d}_{-\mathrm{PGJ}_{2}}$ production in a concentration-dependent manner but did not affect $\mathrm{PGE}_{2}$ production significantly.

Eicosanoids produced by the sequential actions of 5- and 15lipoxygenases (LOXs), promote the resolution of inflammation. The levels of one of these, lipoxin $\mathrm{A}_{4}$, are elevated 2- to 5-fold in FLS cells treated with lenabasum (Zurier et al., 2009). Administration of lenabasum to mice with peritonitis resulted in a $25 \%-75 \%$ decrease in cells invading the peritoneum and caused a 7-fold increase in lipoxin $\mathrm{A}_{4}\left(\mathrm{LXA}_{4}\right)$ measured by mass spectrometry.

The issue of lenabasum purity was addressed (Tepper et al., 2014). In its synthesis, the use of purified starting material and careful purification of the final product resulted in a preparation that was $99.88 \%$ pure. Not taking these precautions can result in the production of byproducts with high affinity for $\mathrm{CB} 1$. The receptor affinity ratio of $\mathrm{Ki} \mathrm{CB} 1 / \mathrm{Ki}$ CB2 was 12.3 for this material. PGJ production in HL-60 cells (est. $\mathrm{ED}_{50}=5 \mu \mathrm{M}$ ) was blocked by the CB2 antagonist SR144528 but not by the CB1 antagonist SR141716. In vivo functional responses in mice, such as catalepsy and hypothermia, with lenabasum that are CB1-mediated were absent at doses up to $30 \mathrm{mg} / \mathrm{kg}$. Thus, it was observed that "conclusions from previous reports on certain actions of ajulemic acid (lenabasum) should now be reconsidered, in particular those claiming psychotropic activity."

Just such a report was made wherein it was stated that lenabasum was not suited for use as a treatment of inflammation in humans because of its CB1-mediated activity (Sumariwalla et al., 2004). No information was given about the source or the purity of the lenabasum used to obtain the data reported. It seems possible that the material contained a significant amount of impurities with high affinity for CB1, making the conclusions about lenabasum not relevant. A detailed response to Sumariwalla et al. (2004) was subsequently published (Burstein and Zurier, 2004). It is worth noting that in none of the human trials with lenabasum, which involved a total of more than 900 subjects up to now, were cannabimimetic actions observed.

A detailed study on the pharmacology of lenabasum in rodents also claimed CB1 mediation for the observed actions (Vann et al., 2007). These included antinociception, catalepsy, hypothermia, and hypomobility in mice as well as a rat model of inflammatory pain. Unfortunately, no information on the purity of the lenabasum was reported, raising serious questions on the significance of their conclusions. The study authors concluded that lenabasum like THC exhibits psychoactive and therapeutic effects at nearly equal doses in these preclinical models. However, when ultrapure preparations of lenabasum were used in these same tests, none of these effects were observed except at doses over 100-fold higher than doses with anti-inflammatory activity. Likewise, the clinical trial findings do not support this assumption (Karst et al., 2003; Salim et al., 2005; Karst, 2007; Spiera et al., 2020).

Lenabasum was found to be effective in a novel model of acute inflammation in which UV-killed Escherichia coli were injected intradermally into healthy humans (Motwani et al., 2018). After a 5-mg twice-daily dose of lenabasum, inhibition of the neutrophil chemoattractant leukotriene $\mathrm{B}_{4}$ was observed; the inhibition of antiphagocytic eicosanoids $\left(\mathrm{PGE}_{2}\right.$, thromboxane $\mathrm{B}_{2}$, and PGF2a) was also reported. At a dose of
$20 \mathrm{mg}$, the biosynthesis of the specialized proresolving lipid mediators, including $\mathrm{LXA}_{4}, \mathrm{LXB}_{4}, \mathrm{RvD} 1$, and RvD3, was stimulated. It was concluded that "for the first time a striking anti-inflammatory and proresolution effects of a synthetic analog of THC in healthy humans" had been demonstrated.

Several publications reported on the effects of lenabasum on PPAR $\gamma$ (Liu et al.,2003; Ambrosio et al., 2007; Gonzalez et al., 2012; Lucattelli et al., 2016). It is important to note that concentrations of lenabasum ranging from 10 to $40 \mu \mathrm{M}$ were required to bind and activate $\operatorname{PPAR} \gamma$, whereas its binding affinity to CB2 is $51 \mathrm{nM}$ (Tepper et al., 2014). Thus, it seems unlikely that PPAR $\gamma$ has an important role in the therapeutic actions of lenabasum.

As a continuation of its development program, Corbus Pharmaceuticals recently completed a phase-3 multicenter, double-blind, randomized, placebo-controlled study assessing the efficacy and safety of lenabasum for the treatment of diffuse cutaneous systemic sclerosis (SSc). Approximately 354 subjects were enrolled in this study at about 60 sites in North America, Europe, Australia, and Asia. The duration of treatment with the study drug was 52 weeks (ClinicalTrials.gov identifier: NCT03398837). Ongoing clinical trials by Corbus also include cystic fibrosis, dermatomyositis, and lupus.

After this history of lenabasum, a stepwise presentation of two proposed mechanisms for the inflammation-resolving action of lenabasum is now given.

\section{COX-Mediated Mechanism for the Resolution of Inflammation}

Cannabinoid Receptor CB2. CB2 is a seven-transmembrane G-protein-coupled receptor (GPCR) that shows binding affinity for several cannabinoids and cannabinoid analogs, including lenabasum (Fig. 1). It is expressed mainly in the periphery, primarily in cells of the immune system (Felder and Glass, 1998). By contrast, the other primary cannabinoid receptor subtype $\mathrm{CB} 1$ is found mainly in the central nervous system, where it mediates the psychotropic action of THC. Activation of CB2 can initiate a series of events eventually resulting in the resolution of chronic inflammation (Fig. 2).

Lenabasum has greater affinity for CB2 over CB1 (Tepper et al., 2014), increasing its usefulness as a clinical agent for humans. Among the various preparations reported, highly purified lenabasum [called JBT-101 in Tepper et al. (2014)] had the weakest affinity for CB1, whereas the original preparation (HU-239) showed the strongest affinity for CB1. The CB1/CB2 ratio of affinities was 12.3 for JBT-101, whereas that for HU-239 was 0.19, a 65-fold difference. Similarly, Dyson et al. (2005) reported a greater affinity for CB1 versus CB2 for a preparation of lenabasum of unstated purity. However, it was recently discovered that impurities can be present in most preparations that account for the additional CB1 activities (Tepper et al., 2014). This is in agreement with the observed CB1-mediated functional responses that were significantly decreased for purified lenabasum compared with HU-239.

Phospholipase A2. Cannabinoid-activated phospholipase A2 (PLA2) promotes the release of free arachidonic acid (AA) from membrane glycerophospholipids (Burstein et al., 1994; Burstein, 2019). AA is a major precursor of eicosanoids, such as the PGs, leukotrienes, and resolvins. Thus far, at least 19 enzymes that possess PLA2 activity have been found in 


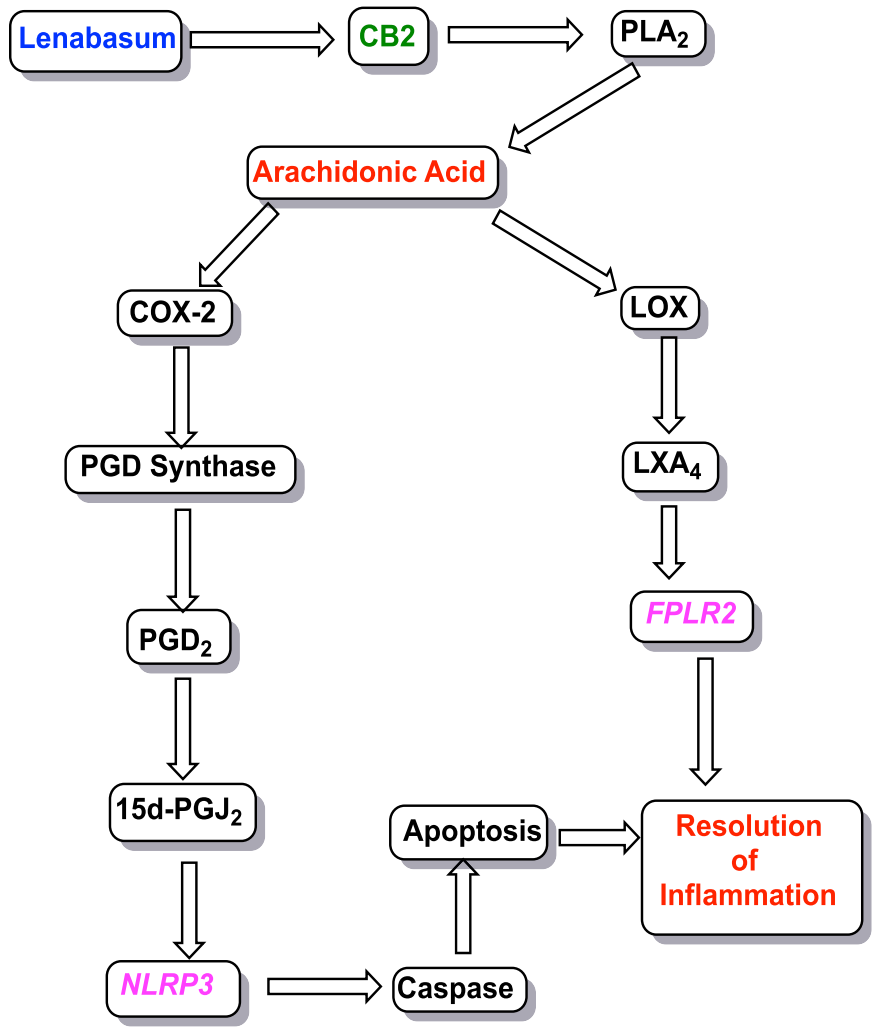

Fig. 2. Mechanisms proposed for the anti-inflammatory actions of lenabasum. Lenabasum binds to and activates the CB2 cannabinoid receptor. Phospholipase activity is then stimulated to promote the release of free arachidonic acid from its phospholipid storage sites. Two pathways, cyclooxygenase- or lipoxygenase-mediated, can then occur, ultimately resulting in the resolution of chronic inflammation. COX-2 action elevates $\mathrm{PGH}_{2}$, which is then followed by PGD synthase action that causes the elevation of $\mathrm{PGD}_{2}$. In turn, this is then rapidly converted in an unregulated process to the cyclopentenone prostaglandin $15 \mathrm{~d}_{-} \mathrm{PGJ}_{2}$. This signaling molecule modulates the activity of the inflammasome neuronal apoptosis inhibitor protein 3 (NLPR3). Caspases are then released that go on to promote apoptosis and resolution of inflammation. The second independent pathway initiated by the actions of 5,12 , and 15 lipoxygenases stimulates the synthesis of the lipoxin $\mathrm{LXA}_{4}$ as well as other eicosanoids. $\mathrm{LXA}_{4}$ reduces IL- $1 \beta$-induced production of the inflammatory cytokines IL- 6 and IL-8 and of matrix metalloproteinases in vitro and in vivo. The eventual result is the resolution of inflammation. FLPR2, formyl peptide receptor 2

mammals (Dennis, 1994). The secretory PLA2 family in which 10 isozymes have been identified consists of low-molecularweight, $\mathrm{Ca}^{2+}$-requiring, secretory enzymes that have a role in a number of biologic processes, such as inflammation, host defense, and atherosclerosis. The cytosolic PLA2 (cPLA2) family consists of three enzymes, among which cPLA2 $\alpha$ plays an essential role in the initiation of AA metabolism. Intracellular activation of cPLA2 $\alpha$ is tightly regulated by $\mathrm{Ca}^{2+}$ and phosphorylation.

Cannabinoids have been reported to modulate the turnover and metabolism of membrane phospholipids in mouse brain preparations through the activation of phospholipases (Hunter et al., 1986). There was a dose-related increase in $\mathrm{PLA}_{2}$ activity leading to an elevation of free AA from phosphatidylcholine. Among the cannabinoids studied was THC-11-oic acid, which was the template molecule used to design lenabasum.

Interestingly, the endocannabinoid anandamide also promotes the release of free AA from phospholipids by activation of $\mathrm{PLA}_{2}$ (Wartmann et al., 1995). Moreover, it was shown that the mitogen-activated protein kinase signal transduction pathway is involved in this response. WI-38 fibroblasts treated with anandamide showed increased mitogen-activated protein kinase activity and increased phosphorylation of the arachidonate-specific cPLA2. Anandamide is a ligand for both CB1 and CB2.

Arachidonic Acid. AA is a key precursor in the synthesis of a number and variety of members of the eicosanoid superfamily of messenger molecules. These include the prostaglandins, the leukotrienes, and the lipoxins, among others, and require free AA for their synthesis. Almost all of the AA in the body is found as a component of various phospholipids and needs to be freed by phospholipase action to be converted to any of the eicosanoids.

One example is seen in the treatment of human FLS cells with lenabasum that caused a robust release of free AA (Stebulis et al., 2008). FLS cells were isolated from synovial tissue or synovial fluid of patients with inflammatory arthritis (four with rheumatoid arthritis, one with psoriatic arthritis). A 1.5- to 2-fold increase in AA release from FLS cells treated with lenabasum was reported in comparison with vehicle-treated controls.

COX-2. COX, whose full name is prostaglandin-endoperoxide synthase, is an enzyme that is responsible for the formation of prostanoids from AA. The specific reaction catalyzed is the conversion of $\mathrm{AA}$ to $\mathrm{PGH}_{2}$ via a transient $\mathrm{PGG}_{2}$ intermediate. There are two types of COX enzymes: COX-1 and COX-2. Both enzymes produce prostaglandins that regulate inflammation, pain, and fever; however, only COX-1 produces prostaglandins that activate platelets and protect the gastrointestinal tract. COX-2 can promote the production of anti-inflammatory prostaglandins, such as the cyclopentenone prostaglandins (CYPGs). NSAIDs inhibit both COX enzymes and reduce synthesis of prostaglandins.

Lenabasum elevated the steady-state levels of COX-2 mRNA in FLS cells (Stebulis et al., 2008). This occurred in a concentration-dependent manner in both unstimulated and $\mathrm{TNF} \alpha$-stimulated cells. A 2 -fold induction of COX-2 was seen 4 hours after stimulation of cells with TNF $\alpha$. However, this was small when compared with the $5-8$-fold induction of COX-2 in cells exposed to lenabasum (Stebulis et al., 2008).

PGD Synthase. Prostaglandin-H2 D-isomerase is an enzyme that in humans is encoded by the prostaglandin-H2 $\mathrm{D}$-isomerase gene. The molecule encoded by this gene is a glutathione-independent PGD synthase that catalyzes the conversion of $\mathrm{PGH}_{2}$ to $\mathrm{PGD}_{2}$. $\mathrm{PGD}_{2}$ plays an important role in inflammatory reactions. It is produced by mast cells, $\mathrm{T}$ helper 2 lymphocytes, and dendritic cells among others (Shimizu et al., 1979).

PGD $_{2}$. The most important prostaglandins found in vivo are $\mathrm{PGE}_{2}$, prostacyclin $\left(\mathrm{PGI}_{2}\right), \mathrm{PGD}_{2}$, and $\mathrm{PGF}_{2 \alpha}$. They are produced in many tissues, and each cell type generates one or two dominant products that can function as autocrine or paracrine lipid mediators to maintain local homeostasis. Prostaglandin production is usually low in uninflamed tissues but increases in acute inflammation, resulting in the recruitment of leukocytes and the infiltration of immune cells.

Through nonenzymic pathways, $\mathrm{PGD}_{2}$ is converted to $\mathrm{PGJ}_{2}$, $\Delta^{12}-\mathrm{PGJ}_{2}, \Delta^{12}-\mathrm{PGD}_{2}, 15$-deoxy- $\Delta^{12,14}-\mathrm{PGJ}_{2}$, or 15 -deoxy- $\Delta^{12,14}-$ $\mathrm{PGD}_{2}$ (Fukushima, 1990). Because of this instability, $\mathrm{PGD}_{2}$ or its metabolites must act in close proximity to the site of their synthesis. $\mathrm{PGD}_{2}$ activates two distinct seven-transmembrane, G-protein-coupled receptors: the D-type prostanoid receptor $\left(\mathrm{DP}_{1}\right)$ and the chemoattractant receptor-homologous molecule expressed on $\mathrm{T}$ helper 2 cells $\left(\mathrm{CRTH} 2\right.$ or $\mathrm{DP}_{2}$ ). Although $\mathrm{DP}_{1}$ and CRTH2/DP 2 share the same ligand, they have little sequence homology. 
15-Deoxy- $\Delta^{12,14}-\mathrm{PGJ}_{2}\left(\mathrm{PGJ}_{2}\right)$ (see Fig. 3A) is a member of the CYPGs (Burstein, 2020). This small group of AA metabolites is characterized by an $\alpha-\beta$ unsaturated keto group that readily undergoes a Michael addition reaction with proteins containing a free $\mathrm{SH}$ group. Thus, they may show a variety of biologic activities in various models, including anti-inflammatory and antiviral effects. No clinical applications for the CYPGs have so far been reported despite their discovery more than three decades ago. This likely is due to their instability when administered in vivo; a stable analog suitable for drug development is apparently not yet available. Thus, an agent that can generate a CYPG in situ close to the site of action may provide a viable approach to this problem in therapeutics. Lenabasum shows promise in doing just that.

$15-$ Deoxy- $\Delta^{12,14}-\mathrm{PGJ}_{2}$ is produced from $\mathrm{PGD}_{2}$ that undergoes a nonenzymic unregulated dehydration reaction to form a new 9,10 double bond to yield a product possessing a cyclopentenone ring. 15-Deoxy- $\Delta^{12,14}-\mathrm{PGJ}_{2}$ has two electrophilic sites at carbons 9 and 13; however, 9 is more electrophilic than 13 and therefore is more active than carbon 9 in forming covalent bonds in transformations involving the Michael addition reaction. This reaction changes the activity of various functionally important target proteins and is a possible mechanism whereby CYPGs may influence cell function. As an example, treatment of FLS cells with lenabasum elevated $\mathrm{PGJ}_{2}$ production in a concentration-dependent manner; however, $\mathrm{PGE}_{2}$ production was not changed significantly (Stebulis et al., 2008).

Regarding mechanism, $\mathrm{I}_{\kappa} \mathrm{B}$ serves to concentrate $\mathrm{NF}_{\kappa} \mathrm{B}$ in the cell cytoplasm, thus preventing it from entering the nucleus and acting as a factor to induce the transcription of genes, many of which are able to regulate inflammatory responses (Scher and Pillinger, 2009). 15-Deoxy- $\Delta^{12,14}-\mathrm{PGJ}_{2}$ forms a covalently bonded adduct with the IKK- $\beta$ subunit of $\mathrm{I}_{\kappa} \mathrm{B}$ kinase, thereby inhibiting the kinases activity and promoting the entry of $\mathrm{NF}_{\kappa} \mathrm{B}$ into the nucleus. This can stimulate the transcription of more than 150 proteins, many of which regulate inflammatory responses. The ultimate effect of this inhibition is to resolve inflammation.

Direct administration of 15 -deoxy- $\Delta^{12,14}-\mathrm{PGJ}_{2}$ into mice with bleomycin-induced lung disease reduced the lung injury, suggesting mediation by this CYPG that is produced by prostaglandin D synthase in this effect (Ando et al., 2003). Intravenous injection of prostaglandin D syntetase (PGDS) cDNA-expressing fibroblasts reduced lung edema, leukocyte infiltration in bronchoalveolar lavage fluid, and pulmonary collagen content at 4 weeks after instillation of bleomycin. This suggests resolution of inflammation. Also, the survival rate in mice injected with PGDS-expressing fibroblasts was higher than that in control mice.

The NLRP3 Inflammasome. Intracellular multiprotein signaling complexes called inflammasomes can initiate a response to pathologic insults in various tissues (Spel and Martinon, 2020). One of these, the NLRP3 inflammasome, is a vital component of the innate immune system, which promotes caspase- 1 activation and the secretion of proinflammatory cytokines IL-1 $\beta / \mathrm{IL}-18$ (Kelley et al., 2019). Activation of the NLRP3 inflammasome has been connected to several inflammatory disorders that include cryopyrin-associated periodic syndromes, Alzheimer disease, diabetes, and atherosclerosis. The eicosanoid $15 \mathrm{~d}_{-} \mathrm{PGJ}_{2}$ and related CYPGs inhibit caspase-1 activation by the NLRP1 and NLRP3 inflammasomes. In a mouse peritonitis model of gout there is a positive response. Using monosodium urate crystals to activate
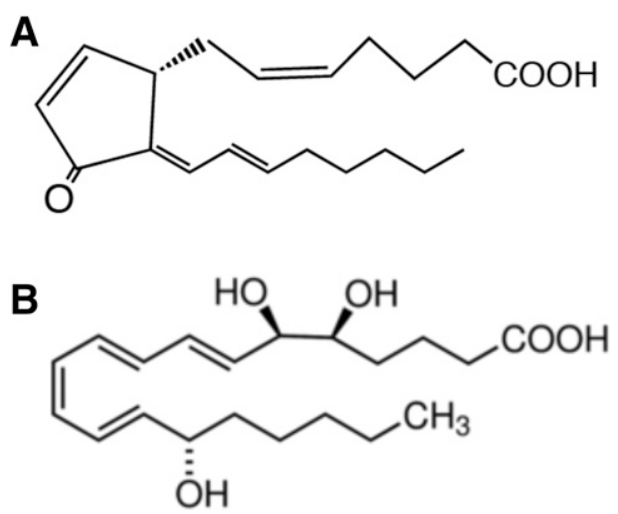

Fig. 3. Chemical structures. (A) $15-$ Deoxy- $\Delta^{12,14}-\mathrm{PGJ}_{2}$ (11-oxo-prosta$5 \mathrm{Z}, 9,12 \mathrm{E}, 14 \mathrm{E}$-tetraen-1-oic acid), molecular mass $=316.4 \mathrm{Da}$, and (B) lipoxin $\mathrm{A}_{4}$ (5S,14R,15S-trihydroxy-6E,8Z,10E,12E-eicosatetraenoic acid), molecular mass $=352.4 \mathrm{Da}$.

NLRP3, PGJ2 caused a significant inhibition of cell recruitment and associated IL- $1 \beta$ release (Maier et al., 2015). Moreover, inflammasome inhibition is independent of $\operatorname{PPAR} \gamma$. For example, an earlier study also demonstrated that $15 \mathrm{~d}$ $\mathrm{PGJ}_{2}$ has anti-inflammatory effects on macrophages that are deficient in PPAR $\gamma$ (Chawla et al., 2001).

In vivo levels of $\mathrm{PGJ}_{2}$ are believed to be in the nanomolar range. So, one may question the physiologic relevance of the micromolar concentrations required for inflammasome inhibition in vitro. However, it has been demonstrated that upon dilution into culture media containing FBS, a majority (97\%-99\%) of the $\mathrm{PGJ}_{2}$ is inactivated by a Michael $1-4$ addition reaction. Therefore, the levels actually available for the cells in vitro are likely at physiologic concentrations. Additionally, it can be argued that systemic measurements of free CYPG in vivo are lower than the actual levels found within localized pockets of inflammation or in the cell vicinity given the high reactivity of these eicosanoids with serum and intracellular proteins (Burstein, 2020).

In a phase 3 trial, one of the unmet needs currently being studied for lenabasum treatment is systemic sclerosis. To provide mechanism data, levels of IL- $1 \beta$ and IL-18 were measured in primary human macrophages that were derived from monocytes (Chintalacharuvu et al., 2020). Lenabasum significantly inhibited IL- $1 \beta$ and IL-18 secretion by monocytederived macrophages, with $\mathrm{IC}_{50}=66.73 \pm 3.92 \mathrm{nM}$ and $349.23 \pm 21.27 \mathrm{nM}$, respectively. MCC950, an inflammasome activation inhibitor, was included as a positive control. The authors concluded that "[l]enabasum inhibits inflammasome activation, which could contribute to potential therapeutic efficacy in SSc and other autoimmune diseases."

Caspase. The caspase family of cysteine proteases can function as primary effectors during apoptosis. They do this by dismantling cellular structures, such as the cytoskeleton, cell junctions, mitochondria, endoplasmic reticulum, Golgi, and the nucleus (Taylor et al., 2008). There are two types of caspases: initiator caspases, such as caspase 2,8,9,10,11,12, and effector caspases, such as caspase 3,6,7. The effector caspases proteolytically degrade many intracellular proteins to carry out a cell death program. There are caspases that can promote both pyroptotic and apoptotic cell death and are activated by inflammasomes (Sagulenko et al., 2018).

Previously it was reported that lenabasum stimulated caspase-3 activity in T lymphocytes (Bidinger et al., 2003). 
At a concentration of $10 \mu \mathrm{M}$, it caused a 9.6-fold increase in enzyme activity. The cells used were human peripheral blood $\mathrm{T}$ lymphocytes that were isolated from healthy volunteers. Positive controls included measurement of caspase- 3 activity in cells treated with $\mathrm{TNF} \alpha$ and camptothecin, known inducers of apoptosis. Lenabasum yielded activity comparable with that of the controls.

Apoptosis. The action called apoptosis is found in multicellular organisms and is a type of programmed cell death. Unlike necrosis, which is a form of traumatic cell death resulting from acute cellular injury, apoptosis is a highly regulated and controlled process. It produces cell fragments called apoptotic bodies that are removed by phagocytic cells. Apoptosis has a role in biologic processes ranging from embryogenesis to aging and is involved in normal tissue homeostasis as well as many human diseases. Apoptosis is characterized by a series of changes to the cellular architecture that contributes to cell death and also prepares cells for removal by phagocytes, thereby preventing adverse immune responses. Several widely used drugs, such as NSAIDs, can alter the levels of apoptosis.

Lenabasum-induced apoptosis was demonstrated in $\mathrm{T}$ cells in a dose- and time-dependent manner using several methods (Bidinger et al., 2003). These were measurement of annexin $\mathrm{V}$ expression, caspase-3 activity, DNA fragmentation, and microscopy. Incubation of cells with $10 \mu \mathrm{M}$ lenabasum caused significant increases of 2.5-8.4 fold in annexin V-positive cells. Caspase-3 activity was also elevated significantly as was DNA fragmentation. In addition, the cells showed shrinkage and blebbing that is a characteristic of apoptosis.

In a separate study, it was reported that apoptosis was induced in RAW 264.7 monocytes that were incubated for 3 days in the presence of the $\mathrm{NF}_{\kappa} \mathrm{B}$ ligand (receptor activator of nuclear factor $\mathrm{NF}_{\kappa} \mathrm{B}$ ligand) (George et al., 2008). Measurement of apoptosis was done using FLOW cytometry (annexin $\mathrm{V}$ and propidium iodide) and measurement of caspases 3 and 8. Cells were incubated in the presence of receptor activator of nuclear factor $\mathrm{NF}_{\kappa} \mathrm{B}$ ligand before $0-30 \mu \mathrm{M}$ lenabasum was added to the media for another 24 hours. Measurement of caspase 3 and 8 activities showed induction of apoptosis by lenabasum when added to differentiated multinucleated cells.

\section{LOX-Mediated Mechanism for the Resolution of Inflammation}

The CB2 and $\mathrm{PLA}_{2}$ steps of the LOX-mediated mechanism for the inflammation-resolving action of lenabasum are the same as in the COX-mediated mechanism in the citation; however, at this point the released AA takes a different pathway in some systems for reasons that are not clear (Fig. 2). An entire new family of eicosanoid signaling molecules is generated, some of which contribute to the resolution of inflammation (Serhan and Samuelsson, 1988; Dalli and Serhan, 2016). These include the lipoxins, resolvins, protectins, and maresins, which are together called specialized proresolving mediators (SPMs).

Lipoxygenases. Lipoxygenases are enzymes that catalyze the formation of dioxygenated hydroperoxides from polyunsaturated fatty acids, such as linolenic acid and arachidonic acid (Mashima and Okuyama, 2015). They are expressed in immune, epithelial, and tumor cells and perform a variety of
TABLE 2

Lenabasum-induced $\mathrm{LXA}_{4}$ synthesis in RAW cells (Burstein et al., unpublished observations).

Culture plates were seeded with $20,000 \mathrm{RAW}$ cells $/ 500 \mathrm{ml}$ media and $50 \mathrm{ml}$ of TNF $\alpha /$ well and incubated for $18 \mathrm{~h}$ at $37^{\circ} \mathrm{C}$. After washing $2 \times$, the cells were treated as shown in the table. The media were then assayed by an $\mathrm{LXA}_{4}$ enzyme immunoassay

\begin{tabular}{lcc}
\hline Treatment $(n=3)$ & Conc. $(\mu \mathrm{M})$ & $\mathrm{LXA}_{4}(\mathrm{ng} / \mathrm{ml})$ \\
\hline Lenabasum & 1.8 & 244 \\
Lenabasum & 5.3 & 266 \\
Lenabasum & 16 & 430 \\
Lenabasum & 48 & 498 \\
Vehicle & - & 187 \\
\hline
\end{tabular}

-, no effect

physiologic actions, which include inflammation, skin disorder, and tumorigenesis. Six LOX isoforms have been identified and characterized: 15-LOX, 15-LOX-2, 12-LOX, 12R-LOX, 5-LOX, and e lipoxygenase-3. 5-LOX activity is tightly controlled by 5 -LOX-activating protein by means of the distribution of 5 -LOX in the nucleus. Lipoxygenase action results in the production of lipoxins that can promote resolution of inflammation (Parkinson, 2006). Of particular relevance to the mechanism of action of lenabasum is $\mathrm{LXA}_{4}$.

Lipoxin $\mathrm{A}_{4}$ (Fig. 3B) is a proresolving mediator that is formed by the sequential actions of 5- and 15-lipoxygenases, which can promote the resolution of inflammation. For example, in mice with peritonitis, dosing with lenabasum resulted in a $69 \%$ decrease in the number of cells invading the peritoneum and a 7-fold increase in $\mathrm{LXA}_{4}$ that was identified by mass spectrometry (Zurier et al., 2009). When added to whole human blood cells, the 12/15 LOX inhibitor baicalein reduced the lenabasuminduced increase in $\mathrm{LXA}_{4}$. $\mathrm{LXA}_{4}$ stimulates its anti-inflammatory proresolving responses via a GPCR termed lipoxin A4 receptor that was identified in human and animal tissues (Serhan and Chiang, 2002). In another system, RAW 264.7 cells, lenabasum also promoted a rise in $\mathrm{LXA}_{4}$ levels (Table 2).

$\mathrm{LXA}_{4}$ is a high-affinity ligand and activator of the FPR2 receptor. This receptor, which is also termed the ALX, ALX/FPR, or ALX/FPR2 receptor, is a GPCR initially identified as a receptor for the leukocyte chemotactic factor $N$-formylmethionineleucyl-phenylalanine based on its amino acid sequence similarity to the known $N$-formylmethionine-leucyl-phenylalanine receptor FPR1. There are no experimental data to support the suggestion that lenabasum-induced $\mathrm{LXA}_{4}$ binds to the formyl peptide receptor 2 receptor. However, this can be considered as a putative event in the inflammation-resolving mechanism of action of lenabasum.

\section{Resolution of Inflammation}

The events described above and depicted in Fig. 2 suggest putative mechanisms for the inflammation-resolving action of lenabasum. There are several reports that lenabasum can resolve chronic inflammation. For example, oral administration of lenabasum reduces joint tissue damage in rats with adjuvant-induced arthritis (Zurier et al., 2003). In another animal model of inflammation, bleomycin-induced scleroderma, it again showed a decrease in pulmonary fibrosis (Gonzalez et al., 2012; Lucattelli et al., 2016).

The results from a phase 2 trial in subjects with scleroderma are in agreement with the preclinical studies (Spiera et al., 2020). 
TABLE 3

Effects of lenabasum on mediators of inflammation

A summary of the changes induced by the actions of lenabasum are shown. They were observed in diverse model systems under a variety of conditions.

\begin{tabular}{lcc}
\hline Report & Inflammation-Resolving & Inflammation-Promoting \\
\hline Zurier et al., 1998 & - & iPGE down \\
Zurier et al., 2003 & - & IL-1 $\beta$ down \\
Parker et al., 2008 & IL-6 down \\
Stebulis et al., 2008 & COX-2 mRNA up & - \\
Stebulis et al., 2008 & Elevated 15d-PGJ & - \\
Bidinger et al., 2003 & Stimulated caspase-3 & - \\
Bidinger et al., 2003 & Annexin V expression & - \\
Bidinger et al., 2003 & DNA fragmentation & - \\
Zurier et al., 2009 & Elevated LXA & - \\
Motwani et al., 2018 & $\mathrm{LXA}_{4}, \mathrm{LXB}_{4}$, RvD1, and RvD3 increased \\
\hline
\end{tabular}

-, no effect; iPGE, immunoreactive PGE

The authors reported that "[g]ene expression in inflammation and fibrosis pathways was reduced and inflammation and fibrosis (was) improved by histological evaluation of skin biopsies from the lenabasum group compared to the placebo group (all $P<0.05$ )."

Further evidence for lenabasum-induced resolution in a novel model of acute inflammation driven by intradermal UV-killed $E$. coli in healthy humans has been reported (Motwani et al., 2018). Lenabasum at a dose of $20 \mathrm{mg}$ stimulated the biosynthesis of specialized proresolving lipid mediators, including $\mathrm{LXA}_{4}, \mathrm{LXB}_{4}, \mathrm{RvD1}$, and RvD3. It was concluded that lenabasum reduces the onset and speeds up the resolution phase of inflammation in this human model.

Suppression of cytokine release during inflammation can result in the resolution of chronic inflammation. Thus, lenabasum has been shown to inhibit the release of the cytokines IL-1 $\beta$ (Zurier et al., 2003) and IL-6, promotors of inflammation. IL- $1 \beta$ release appears to be inhibited by the action of lenabasum more than the other cytokines tested. This is supported by experiments done in human peripheral blood monocytes that were stimulated to release IL- $1 \beta$ by the addition of lipopolysaccharide (Table 2 ). Also, IL- $1 \beta$ release was significantly reduced in cells incubated with lenabasum $\left(10^{-12}\right.$ to $\left.10^{-7} \mathrm{M}\right)$ in vitro or in cells from donors given lenabasum (3-10 mg) orally. Interestingly, $\mathrm{TNF} \alpha$ secretion was not affected under these conditions. IL- $1 \beta$ is a member of the interleukin 1 family of cytokines. The synthesis of the IL- $1 \beta$ precursor is a result of stimulation of innate immune cells by Toll-like receptors or retinoic-acid-inducible gene-like receptors. However, to bind to the IL- 1 receptor, the IL- $1 \beta$ precursor has to be cleaved by a cysteine protease called caspase- 1 that needs to be activated. It is an important mediator of the inflammatory response and is involved in a variety of activities, including cell proliferation, differentiation, and apoptosis.

\section{Summary}

As expressed in Table 3, the resolution of inflammation is needed to restore host cells and tissues to their normal status. In one mechanism, the resolution of acute inflammation is promoted by molecular families called SPMs that include lipoxins, resolvins, protectins, and maresins (Serhan, 2014). The preclinical data reported suggested novel mechanisms involving SPMs, including $\mathrm{LXA}_{4}$, to explain the resolution of inflammation. What occurs is a switch of arachidonic acid-derived prostaglandins and leukotrienes to lipoxins that suppress neutrophil recruitment and induce programmed death by apoptosis (Serhan and Savill, 2005). In a second mechanism, COX-2 action and its products $\mathrm{PGD}_{2}$ and 15-deoxy-PGJ ${ }_{2}$ mediate inflammation-resolving action (Gilroy and ColvilleNash, 2000; Stebulis et al., 2008). The inflammatory response must be actively terminated when no longer needed to prevent damage to healthy tissues. Failure to do so results in chronic inflammation, fibrosis, and cellular destruction. CB2 agonists like lenabasum target both major pathways leading to resolution of inflammation, providing a uniquely effective way to treat chronic inflammatory diseases. In addition, based on these mechanisms it is worth speculating that lenabasum would reduce the so-called "cytokine storms" that occur in other pathologic events that are characterized by severe inflammation.

\section{Acknowledgments}

Thanks are given to Ethan Burstein for comments on the manuscript. Further acknowledgment is given to the University of Massachusetts Medical School for providing the author with office space and nonfinancial support. Much of the data discussed in this review is owed to the outstanding efforts of Robert B. Zurier and Mark Tepper.

\section{Authorship Contributions}

Wrote or contributed to the writing of the manuscript: Burstein.

\section{References}

Ambrosio AL, Dias SM, Polikarpov I, Zurier RB, Burstein SH, and Garratt RC (2007) Ajulemic acid, a synthetic nonpsychoactive cannabinoid acid, bound to the ligand binding domain of the human peroxisome proliferator-activated receptor gamma. $J$ Biol Chem 282:18625-18633.

Ando M, Murakami Y, Kojima F, Endo H, Kitasato H, Hashimoto A, Kobayashi H, Majima M, Inoue M, Kondo H, et al. (2003) Retrovirally introduced prostaglandin D2 synthase suppresses lung injury induced by bleomycin. Am J Respir Cell Mol Biol 28:582-591.

Bidinger B, Torres R, Rossetti RG, Brown L, Beltre R, Burstein S, Lian JB, Stein GS, and Zurier RB (2003) Ajulemic acid, a nonpsychoactive cannabinoid acid, induces apoptosis in human T lymphocytes. Clin Immunol 108:95-102.

Burstein S, Budrow J, Debatis M, Hunter SA, and Subramanian A (1994) Phospholipase participation in cannabinoid-induced release of free arachidonic acid. Biochem Pharmacol 48:1253-1264.

Burstein S, Rosenfeld J, and Wittstruck T (1972) Isolation and characterization of two major urinary metabolites of 1 -tetrahydrocannabinol. Science 176:422-423.

Burstein S and Zurier RB (2004) Pain reduction and lack of psychotropic effects with ajulemic acid: comment on the article by Sumariwalla et al. Arthritis Rheum 50: 4078-4079, author reply 4079-4080.

Burstein SH (2019) Eicosanoid mediation of cannabinoid actions. Bioorg Med Chem 27:2718-2728.

Burstein SH (2020) The chemistry, biology and pharmacology of the cyclopentenone prostaglandins. Prostaglandins Other Lipid Mediat 148:106408.

Burstein SH, Audette CA, Breuer A, Devane WA, Colodner S, Doyle SA, and Mechoulam R (1992) Synthetic nonpsychotropic cannabinoids with potent 
antiinflammatory, analgesic, and leukocyte antiadhesion activities. $J$ Med Chem 35:3135-3141.

Burstein SH, Friderichs E, Kögel B, Schneider J, and Selve N (1998) Analgesic effects of $1^{\prime}, 1^{\prime}$ dimethylheptyl-delta8-THC-11-oic acid (CT3) in mice. Life Sci 63:161-168. Chawla A, Barak Y, Nagy L, Liao D, Tontonoz P, and Evans RM (2001) PPAR-gamma dependent and independent effects on macrophage-gene expression in lipid metabolism and inflammation. Nat Med 7:48-52.

Chintalacharuvu S, Zang P, White B, and Atamas S (2020) Lenabasum, A Cb2 agonist, inhibits inflammasome activation. Ann Rheum Dis 79 (Suppl 1):1372.

Dajani EZ, Larsen KR, Taylor J, Dajani NE, Shahwan TG, Neeleman SD, Taylor MS, Dayton MT, and Mir GN (1999) 1',1'-Dimethylheptyl-delta-8-tetrahydrocannabinol-11-oic acid: a novel, orally effective cannabinoid with analgesic and antiinflammatory properties. J Pharmacol Exp Ther 291:31-38.

Dalli J and Serhan C (2016) Macrophage proresolving mediators-the when and where. Microbiol Spectr 4:10.

Dennis EA (1994) Diversity of group types, regulation, and function of phospholipase A2. J Biol Chem 269:13057-13060.

Dyson A, Peacock M, Chen A, Courade JP, Yaqoob M, Groarke A, Brain C, Loong Y, and Fox A (2005) Antihyperalgesic properties of the cannabinoid CT-3 in chronic neuropathic and inflammatory pain states in the rat. Pain 116:129-137.

Felder CC and Glass M (1998) Cannabinoid receptors and their endogenous agonists. Annu Rev Pharmacol Toxicol 38:179-200.

Fukushima M (1990) Prostaglandin J2--anti-tumour and anti-viral activities and the mechanisms involved. Eicosanoids 3:189-199.

George KL, Saltman LH, Stein GS, Lian JB, and Zurier RB (2008) Ajulemic acid, a nonpsychoactive cannabinoid acid, suppresses osteoclastogenesis in mononuclear precursor cells and induces apoptosis in mature osteoclast-like cells. J Cell Physiol 214:714-720.

Gilroy DW and Colville-Nash PR (2000) New insights into the role of COX 2 in inflammation. $J \mathrm{Mol}$ Med (Berl) 78:121-129.

Gonzalez EG, Selvi E, Balistreri E, Akhmetshina A, Palumbo K, Lorenzini S, Lazzerini PE, Montilli C, Capecchi PL, Lucattelli M, et al. (2012) Synthetic cannabinoid ajulemic acid exerts potent antifibrotic effects in experimental models of systemic sclerosis. Ann Rheum Dis 71:1545-1551.

Hiragata S, Ogawa T, Hayashi Y, Tyagi P, Seki S, Nishizawa O, de Miguel F, Chancellor MB, and Yoshimura N (2007) Effects of IP-751, ajulemic acid, on bladder overactivity induced by bladder irritation in rats. Urology 70:202-208.

Hunter SA, Burstein S, and Renzulli L (1986) Effects of cannabinoids on the activities of mouse brain lipases. Neurochem Res 11:1273-1288.

Karst M (2007) Comments on "cannabimimetic properties of ajulemic acid". $J$ Pharmacol Exp Ther 322:420-421.

Karst M, Salim K, Burstein S, Conrad I, Hoy L, and Schneider U (2003) Analgesic effect of the synthetic cannabinoid CT-3 on chronic neuropathic pain: a randomized controlled trial. JAMA 290:1757-1762.

Kelley N, Jeltema D, Duan Y, and He Y (2019) The NLRP3 inflammasome: an overview of mechanisms of activation and regulation. Int J Mol Sci 20:3328.

Liu J, Li H, Burstein SH, Zurier RB, and Chen JD (2003) Activation and binding of peroxisome proliferator-activated receptor gamma by synthetic cannabinoid ajulemic acid. Mol Pharmacol 63:983-992.

Lucattelli M, Fineschi S, Selvi E, Garcia Gonzalez E, Bartalesi B, De Cunto G, Lorenzini S, Galeazzi M, and Lungarella G (2016) Ajulemic acid exerts poten anti-fibrotic effect during the fibrogenic phase of bleomycin lung. Respir Res $17: 49$

Maier NK, Leppla SH, and Moayeri M (2015) The cyclopentenone prostaglandin 15dPGJ2 inhibits the NLRP1 and NLRP3 inflammasomes. J Immunol 194:2776-2785.

Mashima R and Okuyama T (2015) The role of lipoxygenases in pathophysiology; new insights and future perspectives. Redox Biol 6:297-310.

Mitchell VA, Aslan S, Safaei R, and Vaughan CW (2005) Effect of the cannabinoid ajulemic acid on rat models of neuropathic and inflammatory pain. Neurosci Lett 382:231-235.

Motwani MP, Bennett F, Norris PC, Maini AA, George MJ, Newson J, Henderson A Hobbs AJ, Tepper M, White B, et al. (2018) Potent anti-inflammatory and proresolving effects of anabasum in a human model of self-resolving acute inflammation. Clin Pharmacol Ther 104:675-686.
Parkinson JF (2006) Lipoxin and synthetic lipoxin analogs: an overview of antiinflammatory functions and new concepts in immunomodulation. Inflamm Allergy Drug Targets 5:91-106.

Recht LD, Salmonsen R, Rosetti R, Jang T, Pipia G, Kubiatowski T, Karim P, Ross AH, Zurier R, Litofsky NS, et al. (2001) Antitumor effects of ajulemic acid (CT3), a synthetic non-psychoactive cannabinoid. Biochem Pharmacol 62:755-763.

Sagulenko V, Vitak N, Vajjhala PR, Vince JE, and Stacey KJ (2018) Caspase-1 is an apical caspase leading to caspase-3 cleavage in the AIM2 inflammasome response, independent of caspase-8. J Mol Biol 430:238-247.

Salim K, Schneider U, Burstein S, Hoy L, and Karst M (2005) Pain measurements and side effect profile of the novel cannabinoid ajulemic acid. Neuropharmacology 48:1164-1171.

Scher JU and Pillinger MH (2009) The anti-inflammatory effects of prostaglandins. J Investig Med 57:703-708.

Serhan CN (2014) Pro-resolving lipid mediators are leads for resolution physiology. Nature 510:92-101.

Serhan CN and Chiang N (2002) Lipid-derived mediators in endogenous antiinflammation and resolution: lipoxins and aspirin-triggered 15-epi-lipoxins. ScientificWorldJournal 2:169-204

Serhan CN and Samuelsson B (1988) Lipoxins: a new series of eicosanoids (biosynthesis, stereochemistry, and biological activities). Adv Exp Med Biol 229:1-14

Serhan CN and Savill J (2005) Resolution of inflammation: the beginning programs the end. Nat Immunol 6:1191-1197.

Shimizu T, Yamamoto S, and Hayaishi O (1979) Purification and properties of prostaglandin D synthetase from rat brain. J Biol Chem 254:5222-5228.

Spel L and Martinon F (2020) Inflammasomes contributing to inflammation in arthritis. Immunol Rev 294:48-62.

Spiera R, Hummers L, Chung L, Frech TM, Domsic R, Hsu V, Furst DE, Gordon J, Mayes M, Simms R, et al. (2020) Safety and efficacy of lenabasum in a phase II, randomized, placebo-controlled trial in adults with systemic sclerosis. Arthritis Rheumatol 72:1350-1360.

Stebulis JA, Johnson DR, Rossetti RG, Burstein SH, and Zurier RB (2008) Ajulemic acid, a synthetic cannabinoid acid, induces an antiinflammatory profile of eicosanoids in human synovial cells. Life Sci 83:666-670.

Sumariwalla PF, Gallily R, Tchilibon S, Fride E, Mechoulam R, and Feldmann M (2004) A novel synthetic, nonpsychoactive cannabinoid acid (HU-320) with antiinflammatory properties in murine collagen-induced arthritis. Arthritis Rheum $\mathbf{5 0}$ 985-998.

Taylor RC, Cullen SP, and Martin SJ (2008) Apoptosis: controlled demolition at the cellular level. Nat Rev Mol Cell Biol 9:231-241.

Tepper MA, Zurier RB, and Burstein SH (2014) Ultrapure ajulemic acid has improved CB2 selectivity with reduced CB1 activity. Bioorg Med Chem 22:3245-3251.

Vann RE, Cook CD, Martin BR, and Wiley JL (2007) Cannabimimetic properties of ajulemic acid. J Pharmacol Exp Ther 320:678-686.

Wartmann M, Campbell D, Subramanian A, Burstein SH, and Davis RJ (1995) The MAP kinase signal transduction pathway is activated by the endogenous cannabinoid anandamide. FEBS Lett 359:133-136.

Zurier RB and Burstein SH (2016) Cannabinoids, inflammation, and fibrosis. FASEB J 30:3682-3689

Zurier RB, Rossetti RG, Burstein SH, and Bidinger B (2003) Suppression of human monocyte interleukin-1beta production by ajulemic acid, a nonpsychoactive cannabinoid. Biochem Pharmacol 65:649-655.

Zurier RB, Rossetti RG, Lane JH, Goldberg JM, Hunter SA, and Burstein SH (1998) Dimethylheptyl-THC-11 oic acid: a nonpsychoactive antiinflammatory agent with a cannabinoid template structure. Arthritis Rheum 41:163-170.

Zurier RB, Sun YP, George KL, Stebulis JA, Rossetti RG, Skulas A, Judge E, and Serhan CN (2009) Ajulemic acid, a synthetic cannabinoid, increases formation of the endogenous proresolving and anti-inflammatory eicosanoid, lipoxin A4. FASEB J 23:1503-1509.

Address correspondence to: Sumner Burstein, Department of Biochemistry \& Molecular Pharmacology, University of Massachusetts Medical School, Worcester, MA 01605. E-mail: sumner.burstein@umassmed.edu 